\title{
Thromboembolic Disorders during Pregnancy: Cardiologist Perspective
}

\author{
Anupama V. Hegde ${ }^{1}$
}

\begin{abstract}
Address for correspondence Anupama V. Hegde, MBBS, DNB (Gen Medicine), DNB (Cardiology), Interventional Cardiologist and Assistant Professor Department of Cardiology, M. S. Ramaiah Narayana Heart Centre and Ramaiah Medical college and Hospitals, New BEL Road, MSRIT Post, Bengaluru 560054, Karnataka, India (e-mail: anupamadnb@rediffmail.com).
\end{abstract}

\author{
Abstract \\ Keywords \\ - venous \\ thromboembolism \\ - hematologic changes \\ - hypercoagulable state \\ - pregnancy \\ - anticoagulation
}

The hematologic changes which occur during pregnancy make it a hypercoagulable state. Various factors such as hereditary thrombophilia, obesity, etc. increase the risk of venous thromboembolism in these patients. Though the prevalence is low, it can contribute to significant mortality and morbidity. Deep vein thrombosis is the most common type of venous thromboembolism and pulmonary embolism can sometimes be fatal. Various diagnostic modalities like compression duplex ultrasound of leg veins, echo, ventilation-perfusion scan, and computed tomographic pulmonary angiogram can help in diagnosis. Proper treatment can improve both maternal and fetal outcome.
Safe Motherhood is every woman's earnest dream. Physiological changes which occur during pregnancy as a compensatory mechanism can sometimes become a bane instead of a boon and mar this joyous moment.

\section{Introduction}

Thromboembolic disorders of pregnancy encompass events occurring both during pregnancy and postpartum period. Venous thromboembolic episodes (VTEs) include both deep vein thrombosis (DVT) and pulmonary embolism (PE) and account for $80 \%$ of thromboembolic disorders of pregnancy. DVT is the most common cause of VTE in pregnancy. Approximately $50 \%$ of the events occur during pregnancy and rest during postpartum period. ${ }^{1}$

The prevalence of VTEs during pregnancy is 0.5 to 2.2 per 1,000 deliveries in various studies, ${ }^{2,3}$ and one Indian study has reported that VTE complicates 1 in 1,000 to 2,000 pregnancies and risk during pregnancy and postpartum period to be 0.5 to $3 \%$. VTE can cause significant morbidity and can also be a cause of death in pregnant women. ${ }^{4}$

\section{Hematologic Changes Occurring during Pregnancy}

Pregnancy is associated with significant modification in the coagulation profile. There is increased activity of fibrinogen; clotting factors VII, VIII, X, XII, and VWF (von Willebrand factor); and ristocetin cofactor and decreased activity of protein $S$. Protein C levels remain unchanged, and antithrombin III levels maintain a steady state throughout pregnancy, decrease during labor, and increase again in the postpartum period. All these changes occur due to increased protein synthesis during pregnancy, which is the effect of increasing estrogen levels. All these changes make pregnancy a prothrombotic condition. Activated protein $C$ resistance may occur during pregnancy secondary to increased activity of factor V Leiden and factor VIII with reduced protein $S$ levels. These changes can take up to 6 weeks after delivery to return to nonpregnant levels ( - Table 1 ). ${ }^{5}$

\section{Risk Factors Contributing to Thromboembolic Disorders during Pregnancy}

Various factors contribute to increased risk of VTE during pregnancy. These factors can be preexisting (hereditary or acquired) and/or obstetric causes. Many of the fatal antenatal events can occur during first trimester, with the risk increasing as the gestational age increases. The maximum risk is noted during the first 3 weeks postpartum. Though the absolute risk of VTE in pregnant women is low, it is judicial to take careful history and evaluate the patient with higher risk diligently. Prophylactic anticoagulation needs to be initiated after assessing the risk-benefit ratio. 


\section{Previous Venous Thromboembolic Episodes or Thrombophilia}

Previous VTE increases the risk of recurrent event during subsequent pregnancy by 15 to $25 \%$ with 20 to $50 \%$ of them associated with thrombophilia, predominantly hereditary and few acquired. ${ }^{1}$ Family history of VTE increases the risk of future events in women with asymptomatic thrombophilia. Associated disorders of other coagulation factors or presence of collagen vascular diseases warrant aggressive anticoagulation in patients with thrombophilia and previous VTE. ${ }^{6,7}$

\section{Hereditary Thrombophilia}

Patients with family history of thrombophilia/VTE and type 1 antithrombin deficiency (both activity and antigen are decreased) carry a very high risk when compared with women with heterozygous factor $\mathrm{V}$ Leiden or prothrombin variant. Patients with antithrombin 1 deficiency are resistant to unfractionated heparin (UFH) and may require therapeutic doses of low-molecular-weight heparin (LMWH) throughout pregnancy and for minimum 6 weeks postpartum followed by long-term oral anticoagulation., ${ }^{1,3}$

\section{Antiphospholipid Antibody Syndrome}

Antiphospholipid antibody (APLA) syndrome is the most common cause of acquired thrombophilia and associated with increased risk of VTE during pregnancy. A Canadian study has shown that adjusted odds ratio for PE in these women was 12.9 and for DVT 5.1. These group of patients can have unprovoked or recurrent DVT and require full therapeutic doses of LMWH, followed by long-term oral anticoagulation. ${ }^{1}$ APLA syndrome should be suspected in women with recurrent arterial, venous, or microvascular thrombosis. Obstetric manifestations include recurrent early fetal loss, intrauterine growth retardation, or severe preeclampsia. ${ }^{8}$

\section{Obesity}

Body mass index (BMI) of $>30$ is an independent risk factor for VTE in pregnancy, and overweight (BMI 25-29) women carry lesser risk. However, overweight women also require careful screening.

\section{Age}

Age > 35 years carries a twofold increase in risk of VTE, especially post-delivery. However, a Korean study did not show any additional increase in risk in older women.

\section{Immobility and Long-Distance Travel}

Royal College of Obstetricians and Gynaecologists (RCOG) guidelines consider all long-distance travels ( $>4$ hours) either by road or air to be a risk factor for VTE in pregnancy. A Norwegian case-control study showed that BMI $>25$ and antepartum immobilization amounting to strict bedrest of 1 week or more prior to delivery increased the risk of antepartum or postpartum VTE. ${ }^{9}$

\section{Admission to Hospital}

Admission to hospital for any reason during pregnancy can increase the risk of VTE.
Table 1 Changes in coagulation factors during pregnancy

\begin{tabular}{|l|l|}
\hline Coagulation factors & Change during pregnancy \\
\hline Fibrinogen & Increased \\
VII & Increased \\
VIII & Increased \\
X & Increased \\
XII & Increased \\
VWF & Increased \\
Ristocetin cofactor & Increased \\
Protein S & Decreased \\
Protein C & No change \\
Antithrombin III & No change \\
\hline
\end{tabular}

${ }^{\mathrm{a}}$ Decreases during labor and rises later.

Table 2 Risk factors for venous thromboembolic episode (VTE) in pregnancy

\begin{tabular}{|c|c|c|}
\hline $\begin{array}{l}\text { Preexistent risk } \\
\text { factors }\end{array}$ & $\begin{array}{l}\text { Obstetric risk } \\
\text { factors }\end{array}$ & Other risk factors \\
\hline $\begin{array}{l}\text { Previous history } \\
\text { of VTE }\end{array}$ & $\begin{array}{l}\text { Multiple } \\
\text { pregnancies }\end{array}$ & $\begin{array}{l}\text { Hyperemesis of } \\
\text { pregnancy leading } \\
\text { to dehydration }\end{array}$ \\
\hline Thrombophilia & $\begin{array}{l}\text { Delivery by cae- } \\
\text { sarian section }\end{array}$ & $\begin{array}{l}\text { Treatment of in- } \\
\text { fertility by ovarian } \\
\text { hyper stimulation } \\
\text { using estrogen }\end{array}$ \\
\hline $\begin{array}{l}\text { Antiphospholipid } \\
\text { antibody syn- } \\
\text { drome (persistent } \\
\text { lupus anticoag- } \\
\text { ulant, moderate } \\
\text { to high titers of } \\
\text { anticardiolipin) } \\
\text { antibodies }\end{array}$ & $\begin{array}{l}\text { Postpartum hem- } \\
\text { orrhage requiring } \\
\text { transfusion }\end{array}$ & $\begin{array}{l}\text { Systemic } \\
\text { infections }\end{array}$ \\
\hline $\begin{array}{l}\text { Systemic lupus } \\
\text { erythematosus } \\
\text { (SLE) }\end{array}$ & Prolonged labor & $\begin{array}{l}\text { Prolonged immo- } \\
\text { bilization follow- } \\
\text { ing nonobstetric } \\
\text { surgery }\end{array}$ \\
\hline $\begin{array}{l}\text { Inflammatory } \\
\text { bowel disease }\end{array}$ & $\begin{array}{l}\text { Prolonged } \\
\text { immobilization }\end{array}$ & \\
\hline Malignancies & Still birth & \\
\hline Heart failure & Preterm delivery & \\
\hline Obesity & Preeclampsia & \\
\hline \multicolumn{3}{|l|}{ Multiparity } \\
\hline \multicolumn{3}{|l|}{$\begin{array}{l}\text { Nephrotic } \\
\text { syndrome }\end{array}$} \\
\hline Smoking & & \\
\hline
\end{tabular}

\section{Other Risk Factors}

Comorbidities such as active irritable bowel syndrome, systemic lupus erythematosus (SLE), heart failure, pregnancy-induced hypertension/preeclampsia, hyperemesis, and nonobstetric surgery during antenatal period can all lead to increased risk of VTE during pregnancy (-Table 2 ).

\section{Clinical Features}

Clinical manifestations of DVT and PE are similar to those in nonpregnant women. More than $80 \%$ of women with DVT present 
with pain and swelling of the involved extremity. The difference in calf circumference of $>2 \mathrm{~cm}$ is suggestive of lower limb DVT. Pregnant women with PE can present with acute-onset breathlessness and tachycardia. They can also complain of pleuritic chest pain. Atypical presentations include epigastric discomfort, nausea, vomiting, unexplained hypotension, and undue fatigue. Few of them are asymptomatic and are incidentally picked up during routine antenatal checkup. Massive PE can lead to shock and even cause mortality in pregnant women. ${ }^{10,11}$

\section{Diagnostic Approach to Pregnant Women with Suspected Deep Vein Thrombosis and Pulmonary Embolism}

High index of suspicion is required for diagnosis of PE in pregnant women. Breathlessness can be fallaciously attributed to increase in uterine size causing respiratory compromise or to anemia of pregnancy. Tachycardia, more than the normal physiologic increase in heart rate, demands a more extensive evaluation. Women with prior thromboembolism should be evaluated for thrombophilia and antiphospholipid antibody syndrome.

Elevated levels of D-dimer are commonly seen during the course of pregnancy, and hence loses its significance for diagnosing DVT and PE in pregnant women. A small study has recommended use of modified Well's score, and trimester specific levels of D-dimer can increase the diagnostic accuracy without need for exposure to radiation. ${ }^{12}$

Compression duplex ultrasonography with color Doppler (CUS) is the choice of first line for evaluation of DVT in pregnant women. Use of color Doppler increases the yield of diagnosis as pregnant women tend to have thrombus in the pelvic veins and proximal veins that carry higher risk for pulmonary thromboembolism. This test is safe, lacks ionic radiation, and can be repeated safely in case of initial negative result where the suspicion is high. ${ }^{13}$

\section{Chest X-Ray}

Chest X-ray (CXR) is usually not done routinely during pregnancy. However, chest X-ray posteroanterior (PA) view can be done safely with due precautions for reducing fetal exposure to ionizing radiation when required, to look for evidence of any alternative diagnosis such as pneumonia or pneumothorax. Pulmonary oligemia, enlarged main pulmonary artery, and evidence of wedge-shaped infarcts can be diagnostic of PE. Normal CXR with high index of suspicion warrants further evaluation such as computed tomographic pulmonary angiogram (CTPA) or ventilation-perfusion $\operatorname{scan}(\mathrm{V} / \mathrm{Q} \operatorname{scan}) .{ }^{14}$

\section{Electrocardiogram}

Sinus tachycardia, complete or incomplete right bundle branch block (RBBB), right ventricular (RV) strain, right axis deviation, tall $\mathrm{R}$ in $\mathrm{V}_{1}, \mathrm{~S}_{\mathrm{I}} \mathrm{Q}_{\mathrm{III}} \mathrm{T}_{\mathrm{III}}$, P pulmonale, atrial arrhythmias, and nonspecific ST changes can be suggestive of PE.

\section{Echocardiography}

Two-dimensional (2D) echocardiogram (echo) with color Doppler can be used for prognostication and evaluation of response to treatment. Enlargement of right-sided heart chambers, presence of tricuspid regurgitation, evidence of dilatation of the pulmonary arteries, occasional visualization of thrombus in the pulmonary arteries, and evidence of RV dysfunction can be suggestive or diagnostic of PE. Estimated pulmonary artery pressure suggests the severity of pulmonary hypertension, and serial assessment can help in evaluating the response to therapy. This is noninvasive and very safe in pregnancy.

\section{Magnetic Resonance Venography}

In pregnant women with suspected DVT, compression ultrasound Doppler may be normal. These women tend to have pelvic vein thrombus and carry a high risk of PE. The recently published European Society of Cardiology (ESC) 2018 guidelines suggest that in women with high suspicion in whom initial CUS is negative, magnetic resonance venography could be done to rule out pelvic thrombosis. ${ }^{15}$

\section{Computed Tomographic Pulmonary Angiogram}

Low-exposure computed tomographic pulmonary angiogram (CTPA) can be done safely with low risk of radiation-induced cancer in the fetus and protective shielding of maternal breast tissue to reduce ionizing radiation absorption. CTPA is diagnostic, and a negative scan rules out PE. An alternative diagnosis such as pneumonia can also be made, and unnecessary anticoagulation can be prevented. ${ }^{12,14}$

\section{Ventilation-Perfusion Scan}

$\mathrm{V} / \mathrm{Q}$ scan is not routinely performed due to higher-dose radiation exposure, and $\mathrm{V} / \mathrm{Q}$ scan may yield intermediate and low probability results in patients with high clinical suspicion of $\mathrm{PE}$ and hence is of low diagnostic value. Radiation exposure can be cut down by avoiding ventilation component in patients with normal perfusion and reducing the radiation dose by $50 \%$. Patients with asthma and underlying lung disease are not suitable for $\mathrm{V} / \mathrm{Q}$ scan to diagnose PE. ${ }^{12,14}$

\section{Magnetic Resonance Imaging}

Magnetic resonance imaging (MRI) is safe for both the mother and fetus and can be a good diagnostic aid. Improvements in techniques of MR angiography of pulmonary artery, makes it a single useful diagnostic aid. However safety of usage of gadolinium contrast in pregnant women and its effect on fetus is not well established. ${ }^{14}$

\section{Recommended Imaging Strategy}

Compression duplex ultrasound with Doppler has high sensitivity and specificity in diagnosing DVT. Hence it is recommended as first line of investigation. In pregnant women with negative CUS and high index of suspicion for VTE, magnetic resonance venogram is useful in diagnosing pelvic vein thrombosis.

Risk Stratification of Pregnant Women for Development of Venous Thromboembolic Episodes

The RCOG has suggested a risk stratification module for both ante- and postnatal management in pregnant women. ${ }^{9}$ 


\section{Antenatal Risk Stratification \\ High Risk}

Women with previous episode of VTE not related to surgery are high-risk candidates for development of thromboembolic episode during subsequent pregnancy and require LMWH during antenatal period.

\section{Intermediate Risk}

Women with recent hospitalization, high-risk thrombophilia but no previous VTE, comorbid conditions such as cancer, heart failure, active SLE, inflammatory bowel disease, recent appendicectomy or any other surgical procedure, and ovarian hyperstimulation syndrome carry intermediate risk and may require LMWH during antenatal period.

\section{Low Risk}

Women with less than three risk factors such as obesity (BMI > 30), smoking, age > 35 years, severe varicosities, multiparity, paraplegia, preeclampsia, family history of unprovoked VTE, low-risk thrombophilia, and multiple pregnancy carry low risk. Women with three of the aforementioned risk factors require anticoagulation from 28 weeks of gestation and those with more than four risk factors need anticoagulation from first trimester (-Figs. 1, 2).

\section{Treatment of Acute Deep Vein Thrombosis}

Once diagnosis is established, immediate treatment should be initiated as in-patient..$^{1-3,10}$ No safety studies are available for initiation of treatment on outpatient basis. Anticoagulant of choice is LMWH. UFH can be used, but it carries risk of heparin-induced thrombocytopenia and skin reactions. Vitamin K antagonists such as warfarin are not preferred because of its associated risk of embryopathy.

\section{Dosage of Low-Molecular-Weight Heparin}

Weight gain of pregnancy and altered renal excretion of LMWH mandate that pregnant women should be given high dose of LMWH as against nonpregnant women of same age. For treatment of acute DVT and massive PE monitoring of factor Xa levels, especially during first trimester, is ideal, as recurrence is more common during this period. However, factor Xa assay is not easily available in all centers in India. The recommended dose is twice daily of full-dose enoxaparin or 150 to $200 \mathrm{U} / \mathrm{kg}$ twice daily of UFH. Fondaparinux $7.5 \mathrm{mg}$ can be used in pregnant women with intolerance to heparin compounds in consultation with hematologist.

Anticoagulation therapy should be initiated as early as possible after diagnosis of pregnancy in patients with high risk of developing DVT as prophylaxis. Patients with asymptomatic thrombophilia and no family history of VTE should be closely monitored. After delivery, anticoagulation needs to be continued for 10 days in extremely obese patients (BMI > 40) and those with more than two risk factors. Patients with previous VTE require anticoagulation for at least 6 weeks, and warfarin can be started after 5 days post-delivery with adequate bridging.
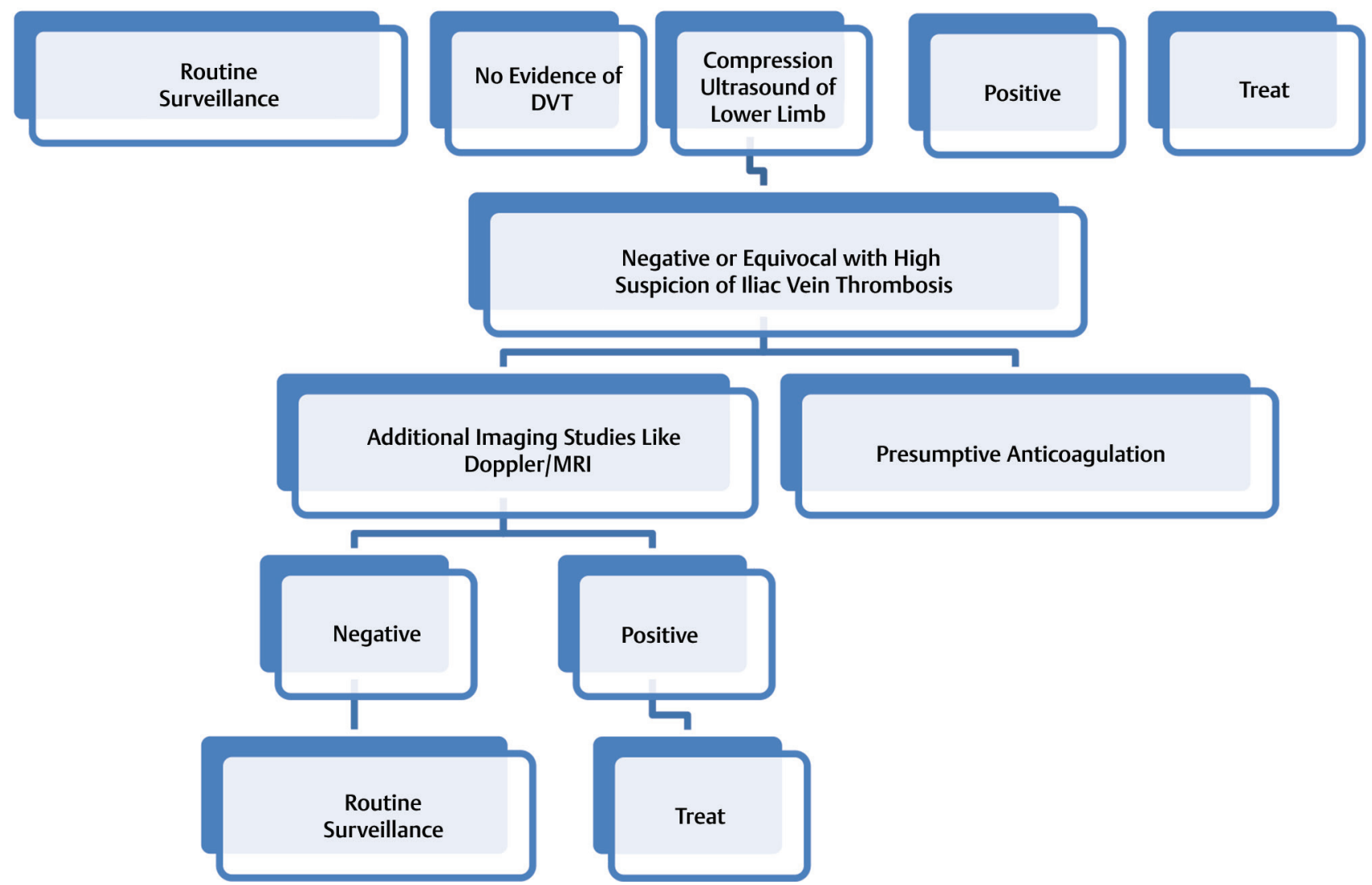

Fig. 1 Algorithm for diagnosis and management of deep vein thrombosis (DVT). MRI, magnetic resonance imaging. 


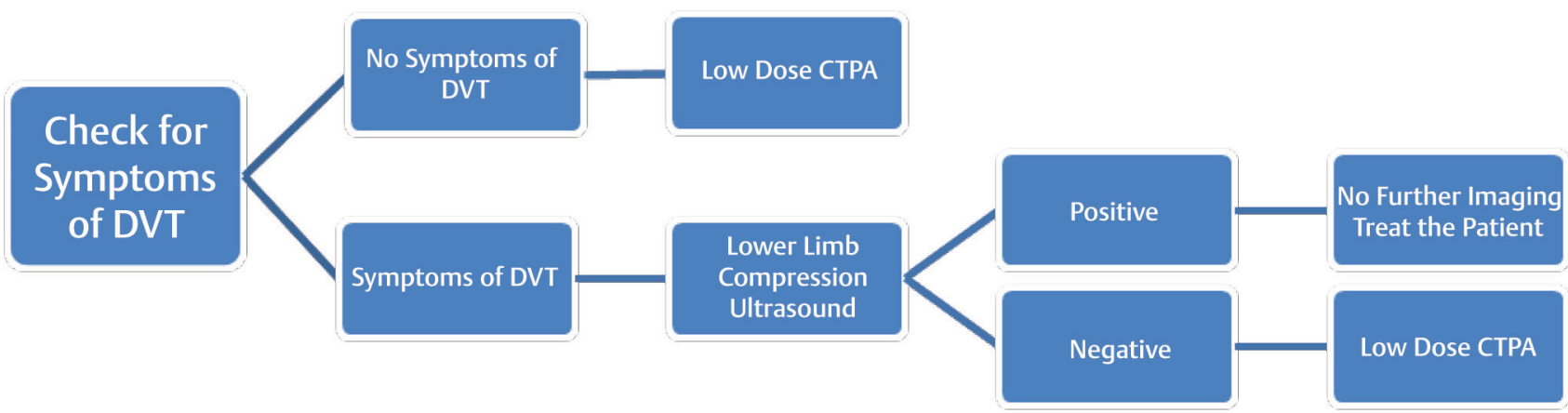

Fig. 2 Algorithm for management of pulmonary embolism. CTPA, computed tomographic pulmonary angiogram; DVT, deep vein thrombosis.

\section{Anticoagulation Therapy during Delivery}

Women on prophylactic or therapeutic anticoagulation should be converted from LMWH to UFH during last month of delivery or as soon as possible when delivery is imminent. ${ }^{1,15}$ For elective delivery, anticoagulation can be stopped 24 hours before induction. Neuraxial blockade can be safely given 12 hours after the last dose of therapeutic dose of LMWH.

Cesarean section increases the risk of VTE by two times. However, routine thromboprophylaxis is not recommended. In patients with increased risk pneumatic compressive stockings can be used. Anticoagulation may be continued after cesarean in women with high risk. Women with antithrombin deficiency can be given antithrombin infusion after delivery.

Women who develop DVT 2 to 4 weeks before delivery are ideal candidates for retrievable venacaval filters. Women with recurrent VTE are also candidates for vena caval filter placement during delivery. ${ }^{16,17}$

It is safer to restart LMWH 4 to 6 hours after vaginal delivery and 6 to 12 hours after cesarean section.

\section{Advantages of Using Low-Molecular-Weight Heparin in Pregnancy ${ }^{15}$}

Advantages of using LMWH in pregnancy include the following: (1) LMWHs have more predictable levels of factor Xa inhibition; (2) reduction in heparin-induced thrombocytopenia that is more common with UFH; (3) regular monitoring of aPTT not required; and (4) lesser incidence of heparin-induced osteopenia and osteoporotic fracture.

\section{Mechanical Devices for Thromboprophylaxis}

In women who have contraindication for anticoagulation, graded compression stockings and intermittent pneumatic compression can be used to prevent DVT in postoperative period.

\section{Management of Pulmonary Embolism}

Pregnant women presenting with acute PE require emergency treatment on in-patient basis. UFH should be started initially, and dose needs to be titrated according to aPTT. Thrombolytic therapy can be given to pregnant women with massive PE and in those presenting with shock. Streptokinase, urokinase, and recombinant tissue plasminogen activator (rTPA) are all safe during pregnancy. They do not cross the placental barrier, and hence risk to the fetus is very low. Post-thrombolysis anticoagulation needs to be continued with LMWH or UFH. In postpartum PE women, LMWH therapy should be bridged with oral anticoagulation until optimal level of anticoagulation is achieved. Safety of usage for newer oral anticoagulants in pregnant and lactating women is not established. ${ }^{15,18}$

\section{Contraception in Women with Venous Thromboembolic Episodes}

Estrogen-containing oral contraceptive pills increase the risk of VTE by many folds, especially those with factor V Leiden deficiency and prothrombin G20210A mutations. Safer options include barrier methods, intrauterine contraceptive devices, and progesterone-only pills.

\section{Other Thromboembolic Disorders}

\section{Cortical Venous Thrombosis}

Cortical venous thrombosis (CVT) is a rare form of VTE but is a cause for stroke in pregnancy. Headache that is the most common feature of CVT can be confused with migraine or preeclampsia. Other symptoms include blurring of vision and vomiting. Papilledema, visual defects, focal neurologic deficits with or without seizures, multifocal neurologic signs, and mental status changes are the usual signs. MRI of the brain with noncontrast magnetic resonance venogram is the investigation of choice. ${ }^{19}$

\section{Superficial Thrombophlebitis}

Superficial thrombophlebitis (ST) is the thrombosis of superficial vein associated with inflammation. The incidence in pregnancy is $0.068 \%$ and may be isolated or secondary to intravenous lines or catheter insertion. Usually self-limiting, but it may require LMWH and nonsteroidal anti-inflammatory drugs (NSAIDs) for 8 to 12 days. Bilateral ST, ST near deep veins such as saphenofemoral junction, systemic infection, and previous DVT can all increase the risk of DVT in these women. ST involving $>5 \mathrm{~cm}$ of the vein can be rarely associated with PE.

\section{Ovarian Vein Thrombosis}

Ovarian vein thrombosis is a rare VTE involving the right ovarian vein in most cases. Its incidence is low (0.05-0.18\%). 
Causative factors include cesarean section, multiple gestation, and infection. Complications include extension into the renal veins, vena cava, and sepsis. These women present with nausea, vomiting, constant lower abdomen or flank pain, guarding, palpable sausage-shaped abdominal mass, fever, chills and rigors, and leukocytosis within 2 weeks of delivery, abortion, or ruptured ectopic. Diagnosis is established by ultrasound of the abdomen, CT scan, or MRI. Treatment is with broad-spectrum antibiotic, and anticoagulation may be required for 4 to 12 weeks.

\section{Arterial Thromboembolism and Stroke}

Arterial thromboembolism, though rare, can be seen in pregnant women with mechanical prosthetic valves receiving inadequate doses of oral anticoagulation and in those with atrial fibrillation..$^{20-24}$ Compared with western counterparts, Indian women are at increased risk due to higher prevalence of rheumatic valvular heart disease. ${ }^{25}$ Management is similar to that in nonpregnant women.

\section{Prevention of Postthrombotic Syndrome}

Postthrombotic syndrome comprises chronic leg swelling, discoloration, pain on walking, or standing occurring in 20 to $40 \%$ of nonpregnant patients who develop a proximal DVT. However, the incidence in pregnant women is unreported, and randomized control studies for management are not available.

\section{Acute Myocardial Infarction}

Acute myocardial infarction (AMI) in pregnant women is rare. However, increase in pregnancies in women aged $>40$ years has increased the risk of atherosclerotic myocardial infarction. The most common cause of myocardial infarction is pregnancy-associated spontaneous coronary artery dissection accounting to $>43 \%$ of the cases. Other causes include thrombus in the coronary arteries (18\%), probable coronary spasm (17\%), and rest due to atherosclerosis..$^{15}$ Conservative management is recommended wherever possible. Recent ESC guidelines recommend primary angioplasty in myocardial infarction (PAMI) in the setting of ST-elevation myocardial infarction (STEMI), with due precautions. Short-term dual antiplatelet therapy is recommended. Statins are contraindicated due to their teratogenic effects on the fetus.

\section{Atheroembolism or Cholesterol Crystal Embolism}

Atheroembolism or cholesterol crystal embolism is extremely rare in pregnant women. They can present with "blue toe" syndrome when there is embolization to distal vessels of the lower limb. Renal artery embolization manifests as severe abdominal pain, nausea, vomiting, and, sometime, with acute kidney injury. Anticoagulation and steroids may be required. Aspirin can be safely used, but statins are contraindicated. Management of these patients requires multidisciplinary approach.

\section{Prepregnancy Counseling}

Women with history of previous thromboembolic episodes and adverse outcomes during previous pregnancies such as recurrent fetal loss need to be thoroughly evaluated; risk-stratified; and counseled regarding recurrence in subsequent pregnancies, need for anticoagulation, and suitable contraceptive methods.

\section{Conclusion}

Thromboembolic disorders during pregnancies, though rare, carry significant risk of morbidity and mortality. Assessment of risk, proper diagnosis, and appropriate management can improve maternal and fetal outcomes. Proper preconceptual counseling and suitable guidelines have made management of these women easy.

\section{Conflict of Interest}

None.

\section{References}

1 Thromboembolism in pregnancy. ACOG Practice Bulletin 2011 Sep;118(3)

2 Bates SM, Middeldorp S, Rodger M, James AH, Greer I. Guidance for the treatment and prevention of obstetricassociated venous thromboembolism. J Thromb Thrombolysis 2016;41(1):92-128

3 Venous Thromboembolism and Antithrombotic Therapy in Pregnancy, SOGC Clinical Practice Guidance, No. 308, June 2014

4 Singh K, Singh MK, Jain S. Venous thrombo-embolism during pregnancy and postpartum period. Nat L J Med Res 2011; 1(1) :16-20

5 Chandra S, Tripathi AK, Mishra S, Amzarul M, Vaish AK. Physiological changes in hematological parameters during pregnancy. Indian J Hematol Blood Transfus 2012;28(3):144-146

6 Croles FN, Nasserinejad K, Duvekot JJ, Kruip MJ, Meijer K, Leebeek FW. Pregnancy, thrombophilia, and the risk of a first venous thrombosis: systematic review and bayesian meta-analysis. BMJ 2017;359:j4452

7 Vora S, Ghosh K, Shetty S, Salvi V, Satoskar P. Deep venous thrombosis in the antenatal period in a large cohort of pregnancies from western India. Thromb J 2007;5:9

8 Garcia D, Erkan D. Diagnosis and management of the antiphospholipid syndrome. N Engl J Med 2018;378(21):2010-2021

9 Reducing the Risk of Venous Thromboembolism during Pregnancy and the Puerperium. RCOG Green-Top Guideline No. 37a, April 2015

10 Thromboembolic Disease in Pregnancy and the Puerperiu Acute Management Green-Top Guideline No. 37b, April 2015

11 McLean KC, James AH. Diagnosis and management of VTE in pregnancy. Clin Obstet Gynecol 2018;61(2):206-218

12 Parilla BV, Fournogerakis R, Archer A, et al. Diagnosing pulmonary embolism in pregnancy: are biomarkers and clinical predictive models useful? AJP Rep 2016;6(2):e160-e164

13 ESHRE Capri Workshop Group. Venous thromboembolism in women: a specific reproductive health risk. Hum Reprod Update 2013;19(5):471-482

14 Pahade JK, Litmanovich D, Pedrosa I, Romero J, Bankier AA, Boiselle PM. Quality initiatives: imaging pregnant patients with suspected pulmonary embolism: what the radiologist needs to know. Radiographics 2009;29(3):639-654 
152018 ESC guidelines for the management of cardiovascular diseases during pregnancy. The Task Force for the Management of Cardiovascular Diseases during Pregnancy of the European Society of Cardiology (ESC). Eur Heart J 2018;00:1-83

16 Gupta S, Ettles DF, Robinson GJ, Lindow SW. Inferior vena cava filter use in pregnancy: preliminary experience. BJOG 2008;115(6):785-788

17 DeYoung E, Minocha J. Inferior vena cava filters: guidelines, best practice, and expanding indications. Semin Intervent Radiol 2016;33(2):65-70

18 Simcox LE, Ormesher L, Tower C, Greer IA. Pulmonary thrombo-embolism in pregnancy: diagnosis and management. Breathe (Sheff) 2015;11(4):282-289

19 McLean K, Cushman M. Venous thromboembolism and stroke in pregnancy. Hematology (Am Soc Hematol Educ Program) 2016;2016(1):243-250

20 Alshawabkeh L, Economy KE, Valente AM. Anticoagulation during pregnancy: evolving strategies with a focus on mechanical valves. J Am Coll Cardiol 2016;68(16):1804-1813
21 Steinberg ZL, Dominguez-Islas CP, Otto CM, Stout KK, Krieger EV. Maternal and fetal outcomes of anticoagulation in pregnant women with mechanical heart valves. J Am Coll Cardiol 2017;69(22):2681-2691

22 Akgüllü Ç, Eryılmaz U, Güngör H. Management of mechanical valve thrombosis during pregnancy, case report and review of the literature. Cemil Zencir Int J Cardiovasc Acad 2017;3:79-82

23 Elkayam U. Anticoagulation therapy for pregnant women with mechanical prosthetic heart valves: how to improve safety? J Am Coll Cardiol 2017;69(22):2692-2695

24 Elkayam U, Goland S, Pieper PG, Silverside CK. High-risk cardiac disease in pregnancy: part I. J Am Coll Cardiol 2016;68(4):396-410

25 Hassouna A, Ammar A, Elnahas Y, Toema A. Limited dose warfarin throughout pregnancy in high-risk patients with mechanical valves: a randomized clinical trial. Hemat Allam Egyptian Heart J 2015;67:115-122 